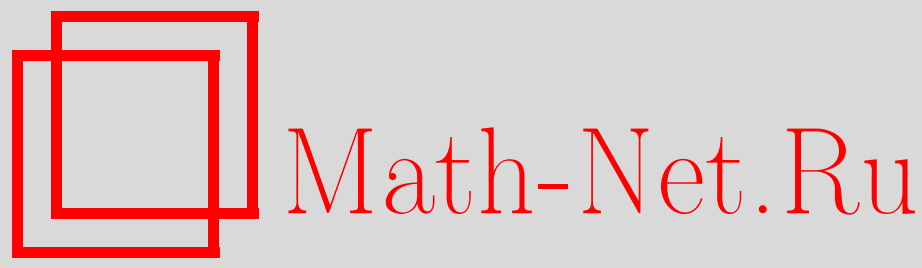

Ф. Багарелло, Свидетельства диссипации квантового гармонического осциллятора с затуханием в контексте псевдобозонов, ТМФ, 2012, том 171, номер 1, 87-95

DOI: https://doi.org/10.4213/tmf6893

Использование Общероссийского математического портала Math-Net.Ru подразумевает, что вы прочитали и согласны с пользовательским соглашением http://www.mathnet.ru/rus/agreement

Параметры загрузки:

IP : 34.229 .108 .108

26 апреля 2023 г., 11:00:42

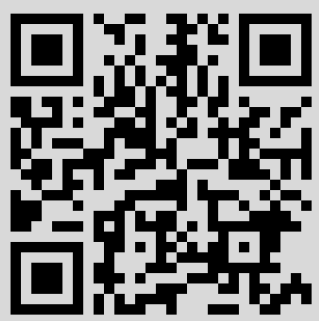




\title{
СВИДЕТЕЛЬСТВА ДИССИПАЦИИ КВАНТОВОГО ГАРМОНИЧЕСКОГО ОСЦИЛЛЯТОРА С ЗАТУХАНИЕМ В КОНТЕКСТЕ ПСЕВДОБОЗОНОВ
}

\begin{abstract}
Известно, что для квантового гармонического осциллятора с затуханием можно определить не зависящий от времени самосопряженный гамильтониан. Показано, что два вакуума, естественным образом связанные с этим оператором, не являются квадратично-интегрируемыми при выражении оператора через псевдобозонные понижающие и повышающие операторы. Этот факт интерпретируется как свидетельство эффекта диссипации классического осциллятора на чисто квантовом уровне.
\end{abstract}

Ключевые слова: квантовый гармонический осциллятор, псевдобозоны.

\section{1. ВВЕДЕНИЕ}

В предыдущей работе [1] мы проанализировали квантовый гармонический осциллятор с затуханием (КГОЗ) в контексте псевдобозонных операторов, которые были недавно введены в работе [2], а затем исследованы нами в ряде работ [3]. Принятая в работе [1] стратегия квантования системы была предложена в работе [4], следующей идее из работы [5], которая восходит к работе Бейтмана [6] и оказалась более всего подходящей для наших целей. Однако хорошо известно, что идея Бейтмана не единственная возможность квантовать диссипативные системы общего положения и, в частности, КГОЗ, существует много разных подходов (подробный список литературы можно найти в работе [7]). Здесь мы лишь напомним, что в квантовой механике диссипативная система довольно часто рассматривается как открытая система, взаимодействующая с некоторым резервуаром, так что, вообще говоря, не требуется, чтобы энергия системы сохранялась, а унитарная группа автоморфизмов, которая представляет динамику замкнутых систем, заменяется некоторой динамической полугруппой.

Возвращаясь к нашим результатам, отметим, что в работе [1] было показано, что даже если в анализе модели естественным образом появляются псевдобозонные операторы, вакуумы двух операторов уничтожения не могут принадлежать гильбертову пространству $\mathcal{H}=\mathcal{L}^{2}\left(\mathbb{R}^{2}\right)$, которому по предположению принадлежит система. Поэтому общая структура, предложенная в работе [3], очевидно не работает.

*DIEETCAM, Università di Palermo, Palermo, Italy. E-mail: fabio.bagarello@unipa.it 
Однако, поскольку детали нашей стратегии вовсе не заданы одним единственным образом, естественно задаться вопросом, существуют ли другие возможности, которые позволили бы построить тот же функциональный формализм, что и в работе [3]. В настоящей работе мы продолжаем этот анализ и отвечаем на этот вопрос.

Работа построена следующим образом: в разделе 2 мы вводим и обсуждаем двумерные псевдобозоны, анализируя некоторые их математические свойства. В разделе 3 мы исследуем КГОЗ, а раздел 4 содержит выводы.

\section{2. ПСЕВДОБОЗОННЫЙ ФОРМАЛИЗМ}

Пусть $\mathcal{H}$ - гильбертово пространство со скалярным произведением $\langle\cdot, \cdot\rangle$ и соответствующей нормой $\|\cdot\|$. Введем две пары операторов $a_{j}$ и $b_{j}, j=1,2$, действующих на пространстве $\mathcal{H}$ и удовлетворяющих коммутационным правилам

$$
\left[a_{j}, b_{j}\right]=\mathbb{1}, \quad\left[a_{1}, a_{2}\right]=\left[a_{1}, b_{2}\right]=\left[a_{2}, b_{1}\right]=0 .
$$

Разумеется, они превращаются в канонические коммутационные соотношения для независимых мод, если $b_{j}=a_{j}^{\dagger}, j=1,2$. Хорошо известно, что $a_{j}$ и $b_{j}$ являются неограниченными операторами, так что их нельзя определить на всем пространстве $\mathcal{H}$. Следуя работе [3] и записывая $D^{\infty}(X):=\bigcap_{p \geqslant 0} D\left(X^{p}\right)$ (общая область определения всех степеней оператора $X)$, рассмотрим следующие допущения.

ДопУщениЕ 1. Существует ненулевое $\varphi_{0,0} \in \mathcal{H}$ такое, что $a_{j} \varphi_{0,0}=0, j=1,2$, $u \varphi_{0,0} \in D^{\infty}\left(b_{1}\right) \cap D^{\infty}\left(b_{2}\right)$.

ДопущЕниЕ 2. Существует ненулевое $\Psi_{0,0} \in \mathcal{H}$ maкое, что $b_{j}^{\dagger} \Psi_{0,0}=0, j=1,2$, $u \Psi_{0,0} \in D^{\infty}\left(a_{1}^{\dagger}\right) \cap D^{\infty}\left(a_{2}^{\dagger}\right)$.

При этих допущениях в прстранстве $\mathcal{H}$ можно ввести следующие векторы:

$$
\varphi_{n, l}=\frac{1}{\sqrt{n ! l !}} b_{1}^{n} b_{2}^{l} \varphi_{0,0}, \quad \Psi_{n, l}=\frac{1}{\sqrt{n ! l !}}\left(a_{1}^{\dagger}\right)^{n}\left(a_{2}^{\dagger}\right)^{l} \Psi_{0,0}, \quad n, l \geqslant 0 .
$$

Теперь определим неограниченные операторы $N_{j}:=b_{j} a_{j}$ и $\mathfrak{N}_{j}:=N_{j}^{\dagger}=a_{j}^{\dagger} b_{j}^{\dagger}, j=1,2$. Можно проверить, что $\varphi_{n, l}$ принадлежит области определения $D\left(N_{j}\right)$ оператора $N_{j}$ и что $\Psi_{n, l} \in D\left(\mathfrak{N}_{j}\right)$ для всех $n, l \geqslant 0$ и для $j=1,2$. Более того,

$$
N_{1} \varphi_{n, l}=n \varphi_{n, l}, \quad N_{2} \varphi_{n, l}=l \varphi_{n, l}, \quad \mathfrak{N}_{1} \Psi_{n, l}=n \Psi_{n, l}, \quad \mathfrak{N}_{2} \Psi_{n, l}=l \Psi_{n, l} .
$$

При таких допущениях, если выбрать нормировку $\Psi_{0,0}$ и $\varphi_{0,0}$ так, что $\left\langle\Psi_{0,0}, \varphi_{0,0}\right\rangle=1$, получим

$$
\left\langle\Psi_{n, l}, \varphi_{m, k}\right\rangle=\delta_{n, m} \delta_{l, k} \quad \forall n, m, l, k \geqslant 0 .
$$

Это означает, что множества $\mathcal{F}_{\Psi}=\left\{\Psi_{n, l}, n, l \geqslant 0\right\}$ и $\mathcal{F}_{\varphi}=\left\{\varphi_{n, l}, n, l \geqslant 0\right\}$ являются биортогональными и поэтому векторы в каждом множестве линейно независимы. Далее предположим следующее.

ДопУЩЕниЕ 3. Множества $\mathcal{F}_{\Psi} u \mathcal{F}_{\varphi}$ полны в $\mathcal{H}$.

Определим операторы $S_{\varphi}$ и $S_{\Psi}$ посредством их действия на базисах $\mathcal{F}_{\Psi}$ и $\mathcal{F}_{\varphi}$ соответственно:

$$
S_{\varphi} \Psi_{n, k}=\varphi_{n, k}, \quad S_{\Psi} \varphi_{n, k}=\Psi_{n, k}
$$


для всех $n, k \geqslant 0$. Отсюда следует, что $\Psi_{n, k}=\left(S_{\Psi} S_{\varphi}\right) \Psi_{n, k}$ и $\varphi_{n, k}=\left(S_{\varphi} S_{\Psi}\right) \varphi_{n, k}$ для всех $n, k \geqslant 0$, поэтому

$$
S_{\Psi} S_{\varphi}=S_{\varphi} S_{\Psi}=\mathbb{1} \quad \Rightarrow \quad S_{\Psi}=S_{\varphi}^{-1}
$$

Другими словами, как $S_{\Psi}$, так и $S_{\varphi}$ обратимы и взаимно обратны. Кроме того, можно также проверить, что оба они - положительные, корректно определенные и симметричные операторы (см. [3]). Более того, по крайней мере формально, можно записать эти операторы в обозначениях "бра-кет":

$$
S_{\varphi}=\sum_{n, k=0}^{\infty}\left|\varphi_{n, k}\right\rangle\left\langle\varphi_{n, k}\left|, \quad S_{\Psi}=\sum_{n, k=0}^{\infty}\right| \Psi_{n, k}\right\rangle\left\langle\Psi_{n, k}\right| .
$$

Эти выражения на данном этапе являются лишь формальными, поскольку ряд может не сходиться в равномерной топологии и операторы $S_{\varphi}$ и $S_{\Psi}$ должны быть неограниченными. Этот аспект всесторонне обсуждался в работе [3] вместе со многими другими свойствами псевдобозонов, которые для нас здесь несущественны.

Интересное наблюдение состоит в том, что эти двумерные псевдобозоны порождают интересные соотношения сплетения среди несамосопряженных операторов (см. [8] и цитируемую там литературу). В частности, легко проверить, что

$$
S_{\Psi} N_{j}=\mathfrak{N}_{j} S_{\Psi}, \quad N_{j} S_{\varphi}=S_{\varphi} \mathfrak{N}_{j}, \quad j=1,2 .
$$

Это связано с тем фактом, что собственные значения, скажем $N_{1}$ и $\mathfrak{N}_{1}$, совпадают и что их собственные векторы связаны операторами $S_{\varphi}$ и $S_{\Psi}$.

\section{3. КВАНТОВЫЙ ГАРМОНИЧЕСКИЙ ОСЦИЛЛЯТОР С ЗАТУХАНИЕМ}

В работе [1] мы рассмотрели КГОЗ в контексте псевдобозонов. Поскольку наша процедура далеко не единственная, отрицательные результаты, полученные нами в работе [1], означают лишь тот факт, что могут возникнуть необычные результаты. Точнее, мы показали, что вакуумы псевдобозонных операторов уничтожения не принадлежат $\mathcal{L}^{2}\left(\mathbb{R}^{2}\right)$. Этот факт, однако, оставляет открытой возможность существования других, более подходящих вариантов, чем те, которые использовались в работе [1], и, в частности, означает, что выбор гильбертова пространства, в котором следует рассматривать модель, был не самым подходящим.

В настоящем разделе мы вкратце приведем результаты работы [1], после чего предложим различные альтернативные подходы. Однако все, что мы предлагаем, ведет к одному и тому же заключению: гильбертово пространство, в котором присутствуют два вакуума псевдобозонных операторов уничтожения, кажется невозможным! Мы прокомментируем этот результат в конце раздела.

3.1. Случай пространства $\mathcal{L}^{2}\left(\mathbb{R}^{2}\right)$. Исходное уравнение движения классического осциллятора с затуханием [5] $m \ddot{x}+\gamma \dot{x}+k x=0$ дополняется вторым виртуальным уравнением $m \ddot{y}-\gamma \dot{y}+k y=0$, и классический лагранжиан системы выглядит KaK

$$
L=m \dot{x} \dot{y}+\frac{\gamma}{2}(x \dot{y}-\dot{x} y)-k x y .
$$


Это соответствует классическому гамильтониану

$$
H=p_{x} \dot{x}+p_{y} \dot{y}-L=\frac{1}{m}\left(p_{x}+\gamma \frac{y}{2}\right)\left(p_{y}-\gamma \frac{x}{2}\right)+k x y,
$$

где $p_{x}=\partial L / \partial \dot{x}$ и $p_{y}=\partial L / \partial \dot{y}-$ сопряженные импульсы. Введение псевдобозонов основано на двух удачных заменах переменных и на каноническом квантовании. Сначала введем новые переменные $x_{1}$ и $x_{2}$ посредством соотношений

$$
x=\frac{1}{\sqrt{2}}\left(x_{1}+x_{2}\right), \quad y=\frac{1}{\sqrt{2}}\left(x_{1}-x_{2}\right),
$$

тогда

$$
\begin{aligned}
& L=\frac{1}{2} m\left(\dot{x}_{1}^{2}-\dot{x}_{2}^{2}\right)+\frac{\gamma}{2}\left(x_{2} \dot{x}_{1}-x_{1} \dot{x}_{2}\right)-\frac{k}{2}\left(x_{1}^{2}-x_{2}^{2}\right), \\
& H=\frac{1}{2 m}\left(p_{1}-\gamma \frac{x_{2}}{2}\right)^{2}+\frac{1}{2 m}\left(p_{2}+\gamma \frac{x_{1}}{2}\right)^{2}+\frac{k}{2}\left(x_{1}^{2}-x_{2}^{2}\right) .
\end{aligned}
$$

Сделаем вторую замену переменных:

$$
\begin{aligned}
p_{+} & =\sqrt{\frac{\omega_{+}}{2 m \Omega}} p_{1}+i \sqrt{\frac{m \Omega \omega_{+}}{2}} x_{2}, & p_{-} & =\sqrt{\frac{\omega_{-}}{2 m \Omega}} p_{1}-i \sqrt{\frac{m \Omega \omega_{-}}{2}} x_{2}, \\
x_{+} & =\sqrt{\frac{m \Omega}{2 \omega_{+}}} x_{1}+i \sqrt{\frac{1}{2 m \Omega \omega_{+}}} p_{2}, & x_{-} & =\sqrt{\frac{m \Omega}{2 \omega_{-}}} x_{1}-i \sqrt{\frac{1}{2 m \Omega \omega_{-}}} p_{2},
\end{aligned}
$$

где мы ввели

$$
\Omega=\sqrt{\frac{1}{m}\left(k-\frac{\gamma^{2}}{4 m}\right)}
$$

и две комплексные величины $\omega_{ \pm}=\Omega \pm i \gamma / 2 m$. Ниже мы будем предполагать, что $k \geqslant \gamma^{2} / 4 m$, т. е. величина $\Omega$ является вещественной. До настоящего момента мы оставались на классическом уровне, так что $\bar{\omega}_{+}=\omega_{-}, \bar{p}_{+}=p_{-}, \bar{x}_{+}=x_{-}$и, следовательно, $\bar{H}_{+}=H_{-}$и $\bar{H}=H$ (см. ниже). Поэтому $H$ является вещественным гамильтонианом. Действительно, с учетом этих определений гамильтониан, по крайней мере формально, выглядит как гамильтониан двумерного гармонического осциллятора

$$
H=\frac{1}{2}\left(p_{+}^{2}+\omega_{+}^{2} x_{+}^{2}\right)+\frac{1}{2}\left(p_{-}^{2}+\omega_{-}^{2} x_{-}^{2}\right)=: H_{+}+H_{-} .
$$

Теперь мы приступаем к каноническому квантованию системы. Следуя работе [4], потребуем, чтобы выполнялись следующие коммутаторы:

$$
\left[x_{+}, p_{+}\right]=\left[x_{-}, p_{-}\right]=i \mathbb{1}
$$

при этом остальные коммутаторы тривиальны. Также потребуем, чтобы выполнялись равенства $p_{+}^{\dagger}=p_{-}$и $x_{+}^{\dagger}=x_{-}$, которые являются квантовым вариантом приведенных выше условий совместности. Теперь появляются псевдобозоны:

$$
\begin{array}{ll}
a_{+}=\sqrt{\frac{\omega_{+}}{2}}\left(x_{+}+i \frac{p_{+}}{\omega_{+}}\right), & a_{-}=\sqrt{\frac{\omega_{-}}{2}}\left(x_{-}+i \frac{p_{-}}{\omega_{-}}\right), \\
b_{+}=\sqrt{\frac{\omega_{+}}{2}}\left(x_{+}-i \frac{p_{+}}{\omega_{+}}\right), & b_{-}=\sqrt{\frac{\omega_{-}}{2}}\left(x_{-}-i \frac{p_{-}}{\omega_{-}}\right) .
\end{array}
$$


Действительно, мы получаем $\left[a_{+}, b_{+}\right]=\left[a_{-}, b_{-}\right]=\mathbb{1}$. Заметим также, что $b_{+}=a_{-}^{\dagger}$ и $b_{-}=a_{+}^{\dagger}$. Более того, гамильтониан $H$ можно записать в терминах операторов $N_{ \pm}=b_{ \pm} a_{ \pm}$как

$$
H=\omega_{+} N_{+}+\omega_{-} N_{-}+\frac{\omega_{+}+\omega_{-}}{2} \mathbb{1},
$$

поэтому гамильтониан КГОЗ несложно записать в терминах псевдобозонных операторов.

В работе [1] мы использовали следующее представление операторов из (10):

$$
\begin{aligned}
x_{+} & =\frac{1}{\Gamma \bar{\delta}-\delta \bar{\Gamma}}\left(\bar{\Gamma} p_{y}+\bar{\delta} x\right), & x_{-} & =-\frac{1}{\Gamma \bar{\delta}-\delta \bar{\Gamma}}\left(\Gamma p_{y}+\delta x\right), \\
p_{+} & =\Gamma p_{x}+\delta y, & p_{-} & =\bar{\Gamma} p_{x}+\bar{\delta} y
\end{aligned}
$$

для всех фиксированных выборов $Г$ и $\delta$ таких, что $\Gamma \bar{\delta} \neq \delta \bar{\Gamma}$. Здесь $x, y, p_{x}$ и $p_{y}-$ попарно сопряженные самосопряженные операторы: $\left[x, p_{x}\right]=\left[y, p_{y}\right]=i \mathbb{1}$. Заметим, что эти операторы также удовлетворяют условиям совместности $p_{+}^{\dagger}=p_{-}$и $x_{+}^{\dagger}=x_{-}$, поэтому естественно представлять $x$ и $y$ как стандартные операторы умножения, а $p_{x}$ и $p_{y}-$ как $-i \partial / \partial x=-i \partial_{x}$ и $-i \partial / \partial y=-i \partial_{y}$. Тогда получаем

$$
\begin{aligned}
& a_{+}=\sqrt{\frac{\omega_{+}}{2}}\left[\left(\beta x+i \frac{\delta}{\omega_{+}} y\right)+\left(\frac{\Gamma}{\omega_{+}} \partial_{x}-i \alpha \partial_{y}\right)\right], \\
& a_{-}=\sqrt{\frac{\omega_{-}}{2}}\left[\left(\bar{\beta} x+i \frac{\bar{\delta}}{\omega_{-}} y\right)+\left(\frac{\bar{\Gamma}}{\omega_{-}} \partial_{x}-i \bar{\alpha} \partial_{y}\right)\right], \\
& b_{+}=\sqrt{\frac{\omega_{+}}{2}}\left[\left(\beta x-i \frac{\delta}{\omega_{+}} y\right)-\left(\frac{\Gamma}{\omega_{+}} \partial_{x}+i \alpha \partial_{y}\right)\right], \\
& b_{-}=\sqrt{\frac{\omega_{-}}{2}}\left[\left(\bar{\beta} x-i \frac{\bar{\delta}}{\omega_{-}} y\right)-\left(\frac{\bar{\Gamma}}{\omega_{-}} \partial_{x}+i \bar{\alpha} \partial_{y}\right)\right],
\end{aligned}
$$

где для упрощения обозначений мы определили $\alpha=\bar{\Gamma} /(\Gamma \bar{\delta}-\delta \bar{\Gamma})$ и $\beta=\bar{\delta} /(\Gamma \bar{\delta}-\delta \bar{\Gamma})$. Заметим, что коэффициенты в (13) удовлетворяют равенствам $\alpha \bar{\delta}=\beta \bar{\Gamma}$ и $\beta \Gamma-$ $\alpha \delta=1$, поскольку эти операторы удовлетворяют псевдобозонным коммутационным правилам.

Согласно равенствам $b_{+}=a_{-}^{\dagger}$ и $b_{-}=a_{+}^{\dagger}$ допущения 1 и 2 тривиализуются, и нам нужно найти только одну квадратично-интегрируемую функцию $\varphi_{0,0}(x, y)$ такую, чтобы прежде всего выполнялось условие $a_{+} \varphi_{0,0}(x, y)=a_{-} \varphi_{0,0}(x, y)=0$. Можно проверить, что решением $a_{+} \varphi_{0,0}(x, y)=a_{-} \varphi_{0,0}(x, y)=0$ является

$$
\varphi_{0,0}(x, y)=N_{\varphi} \exp \left\{-\frac{\beta \omega_{+}}{2 \Gamma} x^{2}+\frac{\delta}{2 \alpha \omega_{+}} y^{2}\right\}
$$

где мы потребовали, чтобы было выполнено равенство

$$
\frac{\omega_{+}}{\omega_{-}}=-\frac{\delta}{\bar{\delta}} \frac{\Gamma}{\bar{\Gamma}}
$$

поскольку тем самым $\varphi_{0,0}(x, y)$ удовлетворяет обоим условиям $a_{+} \varphi_{0,0}(x, y)=0$ и $a_{-} \varphi_{0,0}(x, y)=0$. Разумеется, нам хотелось бы, чтобы функция $\varphi_{0,0}(x, y)$ была квадратично-интегрируемой. Нетрудно проверить, что величины $\beta \omega_{+} / 2 \Gamma$ и $\delta / \alpha \omega_{+}$ 
являются вещественными. Это следует из приведенного выше соотношения между $\omega_{+}$и $\omega_{-}$. Поэтому $\varphi_{0,0}(x, y) \in \mathcal{L}^{2}\left(\mathbb{R}^{2}\right)$, только если $\beta \omega_{+} / \Gamma>0$ и одновременно $\delta / \alpha \omega_{+}<0$. Однако, используя предыдущие соотношения между коэффициентами, получаем

$$
\frac{\beta \omega_{+}}{\Gamma} \frac{\delta}{\alpha \omega_{+}}=\frac{\delta \beta}{\alpha \gamma}=\left|\frac{\delta}{\gamma}\right|^{2}>0 .
$$

Следовательно, функция $\varphi_{0,0}(x, y)$ не может принадлежать пространству $\mathcal{L}^{2}\left(\mathbb{R}^{2}\right)$. Таким образом, мы заключаем, что допущения 1 и 2 не выполнены, так что, по-видимому, из КГОЗ невозможно построить два биортогональных базиса в пространстве $\mathcal{L}^{2}\left(\mathbb{R}^{2}\right)$.

3.2. Замена гильбертова пространства. Однако этот результат не исключает, что выполнение допущений 1 и 2 может быть получено и в другом пространстве $\mathcal{L}^{2}$, например в пространстве подходящего веса. Незамысловатая идея состоит в том, чтобы заменить $\mathcal{L}^{2}\left(\mathbb{R}^{2}\right)$, например, на $\mathcal{H}_{1}:=\mathcal{L}^{2}\left(\mathbb{R}^{2}, e^{-c_{1} x^{2}-c_{2} y^{2}} d x d y\right)$, где $c_{1}$ и $c_{2}$ должны быть положительными константами, выбираемыми таким образом, чтобы волновая функция $\varphi_{0,0}(x, y)(14)$ непременно принадлежала $\mathcal{H}_{1}$. Однако мы немедленно видим недостаток такого подхода: сопряженный оператор в $\mathcal{H}_{1}$ отличается от сопряженного оператора в $\mathcal{L}^{2}\left(\mathbb{R}^{2}\right)$, и это следует учитывать при создании самосогласованной модели. Мы сделаем это в настоящем разделе, в частности покажем, что ожидаемый нами результат вполне разумен, но при этом неверен!

В пространстве $\mathcal{H}_{1}$ скалярное произведение определено как

$$
\langle f, g\rangle_{1}=\int_{\mathbb{R}} d x \int_{\mathbb{R}} d y \overline{f(x)} g(x) e^{-c_{1} x^{2}-c_{2} y^{2}} .
$$

Сопряженный к $X$ оператор $X^{*}$ в $\mathcal{H}_{1}$ определяется равенством $\langle X f, g\rangle_{1}=\left\langle f, X^{*} g\right\rangle_{1}$ для всех $f, g \in \mathcal{H}_{1}$, принадлежащих областям определения операторов $X$ и $X^{*}$ соответственно. Дополнительные подробности и строгие определения, относящиеся к сопряжению неограниченных операторов, можно найти в работе [9]. Легко проверить, что $\partial_{x}^{*}=-\partial_{x}+2 c_{1} x$ и $\partial_{y}^{*}=-\partial_{y}+2 c_{2} y$, что снова дает сопряженный оператор в пространстве $\mathcal{L}^{2}\left(\mathbb{R}^{2}\right)$, если $c_{1}=c_{2}=0$. Взяв в качестве отправной точки операторы $a_{ \pm}$и $b_{ \pm}$, определенные в $(13)$, вычислим их сопряжения в пространстве $\mathcal{H}_{1}$ :

$$
\begin{aligned}
& a_{+}^{*}=b_{-}+\sqrt{2 \omega_{-}}\left(c_{1} x \frac{\bar{\Gamma}}{\omega_{-}}+i c_{2} y \bar{\alpha}\right), \\
& a_{-}^{*}=b_{+}+\sqrt{2 \omega_{+}}\left(c_{1} x \frac{\Gamma}{\omega_{+}}+i c_{2} y \alpha\right), \\
& b_{+}^{*}=a_{-}+\sqrt{2 \omega_{-}}\left(-c_{1} x \frac{\bar{\Gamma}}{\omega_{-}}+i c_{2} y \bar{\alpha}\right), \\
& b_{-}^{*}=a_{+}+\sqrt{2 \omega_{+}}\left(-c_{1} x \frac{\bar{\Gamma}}{\omega_{-}}+i c_{2} y \alpha\right) .
\end{aligned}
$$

Ясно, что эти операторы снова сводятся к сопряженным операторам в $\mathcal{L}^{2}\left(\mathbb{R}^{2}\right)$ при $c_{1}=c_{2}=0$. Также ясно, что потребованные выше условия совместности удовлетворяются только в этом случае: $a_{ \pm}^{*} \neq b_{\mp}$. Тем не менее, если мы продолжим исследование, у нас остается возможность искать решения дифференциальных уравнений 
$a_{+} \varphi_{0,0}(x, y)=a_{-} \varphi_{0,0}(x, y)=0, b_{+}^{*} \Psi_{0,0}(x, y)=b_{-}^{*} \Psi_{0,0}(x, y)=0$. Очевидно, что решение $\varphi_{0,0}(x, y)$ является в точности решением $(14)$, с тем же условием на отношение $\omega_{+} / \omega_{-}$, что и раньше. Волновая функция $\varphi_{0,0}(x, y)$ принадлежит пространству $\mathcal{H}_{1}$, если выполнены неравенства

$$
c_{1}+\frac{\beta \omega_{+}}{\Gamma}>0, \quad c_{2}-\frac{\delta}{\alpha \omega_{+}}>0
$$

Заметим также, что $\varphi_{0,0}(x, y)$ представляет собой собственный вектор оператора $H$ с собственным значением $\left(\omega_{+}+\omega_{-}\right) / 2$. Это может показаться усовершенствованием результатов предыдущего раздела, поскольку и в самом деле существует нетривиальный выбор $c_{1}$ и $c_{2}$, при котором удовлетворяется (16). При любом таком выборе $\varphi_{0,0}(x, y)$ лежит в $\mathcal{H}_{1}$, а также лежит в области определения всех степеней операторов $b_{-}$и $b_{+}$, так что волновые функции $\varphi_{n_{+}, n_{-}}(x, y)$ можно определить формулой (2).

Теперь найдем функцию $\Psi_{0,0}(x, y)$. Согласно (15) получаем

$$
\Psi_{0,0}(x, y)=N_{\Psi} \exp \left\{-\frac{\beta \omega_{+}}{2 \Gamma} x^{2}+\frac{\delta}{2 \alpha \omega_{+}} y^{2}\right\} e^{c_{1} x^{2}+c_{2} y^{2}} .
$$

Эта функция принадлежит $\mathcal{H}_{1}$, если

$$
\frac{\beta \omega_{+}}{\Gamma}-c_{1}>0, \quad c_{2}+\frac{\delta}{\alpha \omega_{+}}<0
$$

Функция $\Psi_{0,0}(x, y)$ представляет собой собственный вектор оператора $H^{*}$, который отличается от $H^{\dagger}=H$, с собственным значением $\left(\omega_{+}+\omega_{-}\right) / 2$. Теперь легко видеть, что эти условия не согласуются с условиями (16): другими словами, невозможно задать $c_{1}$ и $c_{2}$ так, чтобы выполнялись и условия (16), и условия (18). Это означает, что наша исходная простая идея о том, что добавление веса в скалярное произведение в гильбертовом пространстве должно урегулировать ситуацию, не работает так, как мы того ожидали. Более явно, если константы $c_{1}$ и $c_{2}$ удовлетворяют условиям $(16)$, то $\Psi_{0,0}(x, y)$ не удовлетворяет допущению 2. И наоборот, если они удовлетворяют условиям $(18)$, то $\varphi_{0,0}(x, y)$ не удовлетворяет допущению 1 . Поэтому в обоих случаях можно построить только один набор функций в $\mathcal{H}_{1}$, который (скорее всего) является базисом в самом пространстве $\mathcal{H}_{1}$.

3.3. Возможные дальнейшие шаги. Замена гильбертова пространства оказалась интересным упражнением, однако не дала положительных результатов. Поэтому мы вернемся к исходному гильбертовому пространству и рассмотрим четыре оператора $a_{ \pm}$и $b_{ \pm}$, удовлетворяющие соотношениям $b_{ \pm}=a_{\mp}^{\dagger}$ и следующим коммутационным правилам, заданным для удобства только в терминах операторов $a_{ \pm}$и их сопряженных:

$$
\left[a_{+}, a_{-}^{\dagger}\right]=\mathbb{1}, \quad\left[a_{+}, a_{-}\right]=\left[a_{+}, a_{+}^{\dagger}\right]=\left[a_{-}, a_{-}^{\dagger}\right]=0 .
$$

Общие выражения для $a_{+}$и $a_{-}$, которые обобщают соотношения в $(13)$, имеют вид

$$
\begin{aligned}
& a_{+}=\alpha_{x} x+\alpha_{y} y+\beta_{x} \partial_{x}+\beta_{y} \partial_{y}, \\
& a_{-}=\gamma_{x} x+\gamma_{y} y+\eta_{x} \partial_{x}+\eta_{y} \partial_{y},
\end{aligned}
$$


где $\alpha_{x}, \alpha_{y}, \ldots, \eta_{y}$ - комплексные постоянные, которые следует фиксировать. Исходя из вида операторов естественно искать решения уравнений $a_{ \pm} f_{0,0}(x, y)=0$ в виде $f_{0,0}(x, y)=N_{0} e^{-k_{1} x^{2}-k_{2} y^{2}}$ с некоторыми положительными $k_{1}$ и $k_{2}$. Такой выбор гарантирует, что $f_{0,0}(x, y)$ принадлежит не только пространству $\mathcal{L}^{2}\left(\mathbb{R}^{2}\right)$, но и пространству $D^{\infty}\left(b_{+}\right) \cap D^{\infty}\left(b_{-}\right)$. Однако легко проверить, что при таком выборе решения не существует: простые алгебраические преобразования показывают, что $\beta_{x}=\beta_{y}=\eta_{x}=\eta_{y}=0$ и, следовательно, $\alpha_{x}=\alpha_{y}=\gamma_{x}=\gamma_{y}=0$, что невозможно. Поэтому, если мы хотим сохранить вид операторов таким же, как в (20), нужно изменить выражение для $f_{0,0}(x, y)$. Простейшим обобщением является функция $f_{0,0}(x, y)=N_{0} e^{-k_{1} x^{2}-k_{2} y^{2}-k_{3} x y}$, где $k_{3}$ - третья вещественная или комплексная константа, которую следует фиксировать и которая не должна нарушать свойство квадратичной интегрируемости $f_{0,0}$. Можно проверить, что если решение действительно существует, то оно должно соответствовать невещественным значениям величин $\beta$ и $\eta$. Действительно, снова путем простых алгебраических вычислений можно проверить, что решения системы не существует, если $\beta_{x}, \beta_{y}, \eta_{x}$ и $\eta_{y}$ предполагаются вещественными. Кроме того, можно проверить, что при $k_{3}=2 \sqrt{k_{1} k_{2}}$ не существует решения ни при вещественных, ни при комплексных значениях коэффициентов в (20). Другими словами, во всех известных нам ситуациях решения просто не существует, в том смысле, что можно добиться квадратичной интегрируемости, скажем, по $x$, но не по $y$, или наоборот. Или же можно добиться квадратичной интегрируемости как по $x$, так и по $y$ для функции $\varphi_{0,0}(x, y)$, но не для второй волновой функции $\Psi_{0,0}(x, y)$. Кроме того, ясно, что изменение определений в $(20)$ опасно, поскольку может нарушить условия совместности или коммутационные правила, или и то, и другое. Аналогично, изменяя выражение для $f_{0,0}(x, y)$, легко нарушить справедливость допущения 1: наши варианты представляются единственно возможными!

На первый взгляд, полученные результаты представляются неудовлетворительными. С другой стороны, мы полагаем, что они в высокой степени физически мотивированны. Действительно, вспомним, с чего мы начали: наша вещественная система - гармонический осциллятор с затуханием - взаимодействовала с виртуальным (вынуждаемым) гармоническим осциллятором. Поэтому на классическом уровне, когда первый осциллятор терял энергию, она переходила ко второму таким образом, что система в целом оставалась консервативной. Существование такого классического потока энергии на квантовом уровне означает невозможность получить одновременно функции $\varphi_{0,0}$, и $\Psi_{0,0}$ в одном гильбертовом пространстве: например, если функцию $\varphi_{0,0}$ можно нормировать, то нельзя нормировать функцию $\Psi_{0,0}$, ее норма слишком сильно возрастает. При этом представляется, что данный результат не удастся изменить: если мы изменим гильбертово пространство, чтобы включить большее число функций, то окажется, что мы так или иначе выйдем из пространства, поскольку нам все равно надо изменить сопряжение. Можно искать другие, возможно более удобные, представления псевдобозонных операторов. Однако требование того, чтобы их вакуумы были квадратично-интегрируемыми, приводит к набору алгебраических уравнений, вообе не имеющих решений. Нет нужды говорить, что наш выбор не охватывает все возможные ситуации, так что могут быть обнаружены другие, довольно специальные решения, отражающие построения из раздела 2. Однако мы утверждаем, что эти решения не могут существовать, именно вследствие неконсервативной природы нашего осциллятора. 


\section{4. ЗАКЛЮЧЕНИЕ}

В настоящей работе более детально рассмотрен КГОЗ, который уже обсуждался в работе [1]. Показано, что работа в различных гильбертовых пространствах или изменение представления псевдобозонных лестничных операторов не меняет основных результатов нашего предыдущего анализа, а именно того факта, что не все формальные собственные состояния операторов числа частиц являются квадратично-интегрируемыми. Этот факт мы считаем связанным с неконсервативной природой анализируемой квантовой системы.

Благодарности. Автор благодарит Ministero dell'Istruzione, dell'Università e della Ricerca за финансовую поддержку.

\section{Список литературы}

[1] S. T. Ali, F. Bagarello, J. P. Gazeau, J. Math. Phys., 51:12 (2010), 123502, 11 pp., arXiv: 1010.4221.

[2] D. A. Trifonov, "Pseudo-boson coherent and Fock states", Proceedings of the 9th International Workshop "Complex Structures, Integrability and Vector Fields" (August 25-29, 2008, Sofia), eds. K. Sekigawa, V. Gerdjikov, S. Dimiev, World Scientific, Singapore, 2009, 241-250, arXiv: 0902.3744.

[3] F. Bagarello, J. Math. Phys., 51:2 (2010), 023531, 10 pp., arXiv: 1001.1136; 51:5 (2010), 053508, 12 pp., arXiv: 1003.4620; Phys. Lett. A, 374:37 (2010), 3823-3827, arXiv: 1007.4349 .

[4] R. Banerjee, P. Mukherjee, J. Phys. A, 35:27 (2002), 5591-5598, arXiv: quant-ph/0108055.

[5] H. Feshbach, Y. Tikochinsky, Trans. N. Y. Acad. Sci., 38 (1977), 44-53.

[6] H. Bateman, Phys. Rev., 38:4 (1931), 815-819.

[7] D. Chruściński, J. Jurkowski, Ann. Phys., 321:4 (2006), 854-874, arXiv: quant-ph/0506007.

[8] F. Bagarello, J. Phys. A, 43:17 (2010), 175203, 11 pp., arXiv: 1003.2288.

[9] М. Рид, Б. Саймон, Методъ современной математической физики, т. 1: Функииональный анализ, Мир, М., 1977.

Поступила в редакцию 2.04.2011, после доработки 12.05.2011 